\title{
SEMSIM POWER AS AN APPLICATION OF USES
}

\author{
David Ciechanowicz \\ TUM CREATE \\ Singapore \\ email: david.ciechanowicz@tum-create.edu.sg \\ Heiko Aydt \\ TUM CREATE \\ Singapore \\ Alois Knoll \\ email: heiko.aydt@tum-create.edu.sg \\ Robotics and Embedded Systems \\ Technische Universität München \\ Munich, Germany \\ email: knoll@in.tum.de
}

\begin{abstract}
With the increasing complexity of real-world energy systems its modeling process becomes even more crucial when large-scale simulations are conducted. Being computational intensive and therefore requiring efficient simulation models a modeling scheme with a well-defined formal syntax definition is developed and together with its meta model proposed in this paper. This Universal Scheme for modeling Energy Systems (USES) is the preferred language for the power system simulation presented here as being part of our Scalable Electro-Mobility Simulation (SEMSim) platform. For investigating the impact of electro-mobility on the city infrastructure the transmission system of Singapore is described as real, data, and formal model, the first two based on USES.
\end{abstract}

\section{KEY WORDS}

Power System Simulation, Modelling Language

\section{Introduction}

Energy systems are networks in which extraction, refinement, conversion, transportation, distribution, and utilization of different forms of energy take place to provide a set of services. An energy system involves multiple interconnected energy chains that provide a certain energy service. These energy chains are, to some extent, competing with each other as there may be multiple possible chains that lead to the provision of the same energy service. Figure 1 shows an example of an energy chain which consists of a sequential series of linked stages, alternating commodities (e.g., energy goods) and processes (e.g., energy conversions).

Energy systems can be of paramount complexity, especially on the large-scale with a huge amount of interconnected energy chains. Traditional architectures of power systems, which are specialized variants of an energy system that are only considering energy chains involving electrical energy, reaches its limits. They are thus succeeded by smart grids with diverse, intermittent and decentralized en-

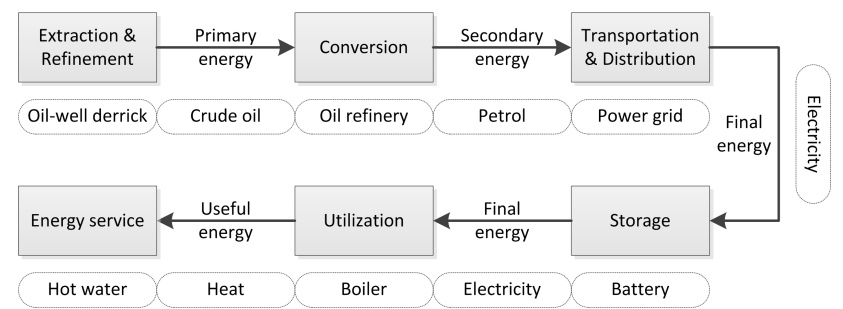

Figure 1. Example of an energy chain from the extraction to the provision of energy services.

ergy sources to form distributed energy systems which are controlled in a decentralized and autonomous fashion [1]. Simulation is needed in order to understand and plan the functionality of complex energy systems based on computational models.

Large-scale simulations of complex energy systems are compute intensive and thus require efficient simulation models. The most common existing modeling scheme, the extended Reference Energy System (eRES) approach [2], gives a simplified image of the reality in a conceptual and representational way. Its syntax, however, is inconsistent, complex, and bears a lot of exceptions. We thus propose the Universal Scheme for modeling Energy Systems (USES), an alternative language with a simple graphical representation and a well-defined syntax.

Having a clear and well-defined formal syntax definition of a language offers several advantages. From an implementation perspective, simulation models can be easily verified against the formal specification. Furthermore, a standardized method for modeling energy systems can be established which facilitates interoperability of different simulation models and exchange of models and data between different research groups. Another advantage of having a formal specification is the ability to easily generate synthetic power systems that can be used for simulationbased what-if scenario analysis.

Scenario space exploration by means of simulation are very time consuming, in particular when not done automatically. Manual generation of simulation scenarios may 
involve several steps to describe and implement an energy system model. Alternatively, automatic generation of energy systems with certain properties, representing different scenarios and their assumptions, can significantly increase the number of scenarios that can be evaluated. We are currently working towards a Scalable Electro-Mobility Simulation (SEMSim) platform which will facilitate coupling of an agent-based traffic simulation [3] with an agent-based power system simulation (e.g., [4]) to study the impact of electro-mobility on the power system on the scale of an entire city.

In the application context of SEMSim, exploratory simulation plays an important role as it will allows us to evaluate many electro-mobility scenarios. In order to automatically explore a scenario space in which different scenarios may also be concerned with different power system configurations, it is necessary to be able to automatically generate a simulation model of the power system that reflects the scenario. For this purpose, automatic model generation based on USES will be employed.

In this paper we describe SEMSim Power, the power system simulation of SEMSim, as an application of USES. This paper is structured as follows. In Section 2 we discuss related work on the topic of modeling energy systems. We introduce USES in Section 3 and describe its application in the context of SEMSim in Section 4. We present a summary and our conclusions in Section 5.

\section{Related Work}

For investigating real-world energy systems a modeling scheme is needed that provides a simplified image of the system in a conceptual precise way. Different languages exist that may be used for creating an energy system model. These languages can be differentiated into two classes, languages that were specifically designed for this purpose and languages that were not.

Among the latter one are system dynamics models [5], the precedence and block diagram methods [6], process chains [7], one-line diagrams [8], and petri nets [9]. Hypergraphs [10] may also be an option, especially when the paradigm change of energy systems from a system to a system-of-systems occurs as described in [11]. These methods all have in common that they were not especially designed for modeling energy systems and are therefore either conceptually too complex or lack the necessary expressiveness.

The Reference Energy Systems (RES) concept proposed by [12] and further described in [13] was the first unitary modeling scheme specifically designed for the purpose of modeling energy systems that represents the topology of the investigated energy system. Not being able to cope with the modeling requirements of complex energy systems the extended RES (eRES) was proposed by [2].

eRES has an inconsistent and complex formal syntax specification with an unnecessary high amount of exceptions. It therefore seems antiquated when it comes to mod- eling future distributed, adaptive, and multidisciplinary energy system-of-systems with intermittent and decentralized energy sources [1][11]. To understand, investigate, optimize, and plan the functionality of such complex energy systems, computer simulations are needed which are based on computational models. The nodal admittance matrix, or Y Matrix, is the most used tool for algorithms optimizing power systems [8].

The analysis of power systems and therefore power system simulation software can be divided into the classes load flow, short circuit, transient stability, unit commitment, and optimal power flow which are described in detail in [14]. It is due to the diversity that no simulation can respond to the needs of all of the different classes, especially not when it comes to simulating smart grids with an additional communication capability.

The development of a comprehensive and powerful power system simulation, even only in one of the classes, is complex and time-consuming and mainly results in commercial applications. Non-commercial simulations for academic research mostly lack the necessary comprehensiveness because they are only actively developed by a tiny group of people. A comprehensive list of 23 commercial and 12 non-commercial power system analysis software products was created ${ }^{12}$.

Existing literature mainly reveals the four different non-commercial open-source power system simulations GridLAB-D ${ }^{34}[15]$, InterPSS ${ }^{5}[16]$, OpenDSS $S^{6}$, and $P S A T^{7}$. A short description of the latter three and some of the other non-commercial simulation tools is given in [17]. GridLAB-D and OpenDSS are not only described in more detail in [18] but also utilized for two case studies.

\section{USES}

The syntax of the most common existing modeling scheme, the extended Reference Energy System (eRES) approach [2], is inconsistent, complex, and includes various exceptions. The Universal Scheme for modeling Energy Systems (USES), which is introduced in the remainder of this section, is a language that has been developed with the shortcomings of eRES in mind. As such it represents an alternative to eRES that does not come with the same disadvantages.

\footnotetext{
${ }^{1}$ http: / / www. openelectrical .org/wiki/index.php? title=Power_Systems_Analysis_Software $\backslash$ \#ETAP

${ }^{2}$ http://ewh. ieee.org/cmte/psace/CAMS_ taskforce/software.htm

${ }^{3}$ http: //www.gridlabd.org/

${ }^{4}$ http: //sourceforge.net/apps/mediawiki/ gridlab-d/index.php?title=Publications

5 http: //www.interpss.org/

6 http://www. smartgrid.epri.com/SimulationTool. aspx

${ }^{7}$ http://www3. uclm. es/profesorado/federico. milano/psat.htm
} 


\subsection{Conceptual and Representational Description}

The conceptual description of USES can be divided mainly into two parts: commodities and processes. A commodity is a material stream or set that is quantifiable and that is produced and/or consumed by processes. Each commodity is of a specific type only allowing a connection between two processes capable of providing or consuming this commodity type. The connection is directed without having to introduce dedicated process interfaces. Figure 2 shows a graphical representation of a commodity.

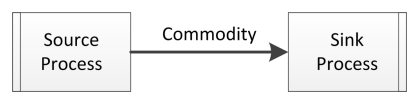

Figure 2. A commodity connecting two processes.

A process is a representation of a physical device which transforms commodities into other commodities. As shown in Figure 3, we further distinguish between source, intermediate, and sink processes. Every energy chain modeled in USES has to start with a source process having no inputs but only outputs (e.g., energy harvesting or generating) and has to end with a sink processes having no outputs but only inputs (e.g., energy consuming). Intermediate processes can have multiple inputs and outputs and therefore be both, consumers and producers of energy.

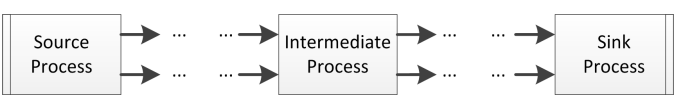

Figure 3. Source process (a), intermediate process (b) and sink process (c).

It is possible to aggregate multiple processes into aggregated processes. Aggregated source, intermediate, and sink processes behave exactly as their non-aggregated counterparts in relationship to other elements on the same hierarchical level. Instead of describing the input-output transformations in form of mathematical equations it hosts an energy sub-system on a hierarchical below lying layer regarding content. The use of aggregated processes within USES is transparent and should be done when mathematical equations are not sufficient for describing their function. Their graphical representation is illustrated in Figure 4.

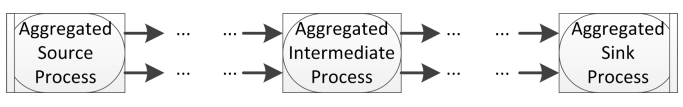

Figure 4. Aggreated source process (a), aggregated intermediate process (b) and aggregated sink process (c).

When representing bi-directional commodity flows the concept of having directed connections, where inputs are connected to the left and outputs are connected to the right of a process, quickly becomes cumbersome. By introducing an alternative graphical representation without having to change the conceptual definition of USES, commodities may also be drawn as bi-directional connections. The graphical representation shown in the Figures 5 and 6 are therefore conceptually equal.

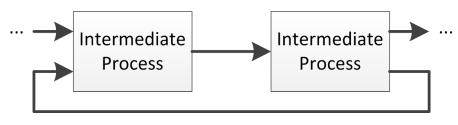

Figure 5. Directed commodities.

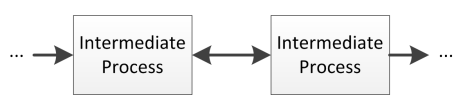

Figure 6. Bi-directional commodities.

\subsection{Meta Model}

The concepts of USES and their syntactical relationships among each other follow certain rules. In this section a meta model of USES is created and presented. The UML class diagram language is applied because it is a widelyused and regarding its syntax fully formally specified language $^{8}$.

The three main classes USES model, Process, and Commodity as well as their attributes and relationships among each other are shown in the upper half of Figure 7. The inheritance structure of processes as well as the associations among the specialized sub-classes is shown in the bottom half of the figure. Relationships are denoted in the multiplicity-notation of the UML class diagram language.

Only attributes that are mandatory for the definition of the syntax of USES are part of the meta model. Among them are the id (unique number identifying every element), the shortName (name identifying every element in its graphical representation), the scope (the id of the aggregated process or USES model the element is part of on the hierarchical above lying layer), and the idCommodityType (unique number for every different type of commodities).

Attributes and relationships are only illustrated once on the most generalized level and not repeated on lower specialized levels according to the general concept of inheritance [19]. Besides inheritance, compositions and associations are also used among the depicted classes and illustrated in Figure 7. Any association is assumed to be bi-directional. Classes whose names are written in italic are abstract classes according to the UML standard. They cannot be instantiated and, as already stated in Section 3.1, do not have any graphical representation.

\footnotetext{
${ }^{8}$ http: //Www.omg.org/spec/UML/2 .5/Beta1/PDF /
} 


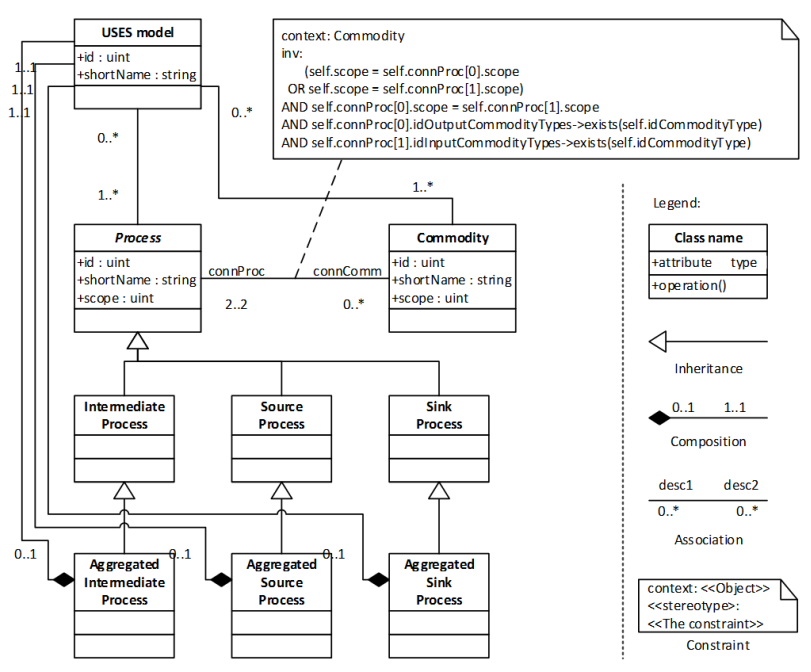

Figure 7. Meta model of USES in the class diagram language of the UML.

The constraint in the relationship between the two classes Process and Commodity is annotated as an invariant using the formal Object Constraint Language (OCL) ${ }^{9}$. OCL is one language for constraint specifications within class diagrams to depict syntactical rules which cannot be modeled using explicit diagrammatic language constructs.

\section{Application: SEMSim Power}

In the context of electro-mobility research conducted at TUM CREATE in Singapore, we are developing the Scalable Electro-Mobility Simulation (SEMSim) platform [3]. Within this framework multiple simulation federates are coupled by means of using the High Level Architecture (HLA) [20]. The two main federates, an agent-based traffic simulation (SEMSim Traffic) and an agent-based power system simulation (SEMSim Power), will be used to investigate the impact of electro-mobility on the city infrastructure. Figure 3.2 illustrates the coupling of the traffic simulation and the power system simulation.

The traffic system and the power system affect each other by means of charging and discharging of vehicle batteries at charging stations distributed across the city. Depending on the charging station distribution and the dynamics of the traffic participants in terms of charging behavior, we intend to investigate the dynamics of energy demand in various electro-mobility scenarios. These studies are mainly concerned with establishing a better understanding of how disruptive city-wide electro-mobility (several 10k electric vehicles) may be.

In this paper, we focus on SEMSim Power, an agentbased power system simulation. The various entities in this simulation are modeled as intelligent autonomous agents, as described in [4]. SEMSim Power is implemented in

${ }^{9}$ http://www.omg.org/spec/ocL/
Java, using a MySQL database for storing model-related data. The simulation model of SEMSim Power is based on the real model and the data model provided by USES. The formal model describes the behavior of the modeled entities. Separating the real model, the data model and the formal model is advantageous because different formal models and quantitative data may be specified for the same real model without the need for modifications. The basic description of the three different model types and their application in SEMSim Power is explained in the remainder of this section.

\subsection{Real Model}

The real model of SEMSim Power is based on processes and commodities as USES and qualitatively describes the flow of energy from the energy sources to its final use. Hence, it describes the topology or the structure of the modeled energy system. It is free of quantitative data or any behavior of the modeled entities. Therefore, only nonquantifiable objectives can be investigated.

The real model of SEMSim Power is illustrated in Figure 9 using the graphical USES representation. To enhance the overview only elements on the most aggregated level are visible, commodity labels are spared out. The model consists of five power station congeries (PSC1 to PSC5) as aggregated source processes where each one contains a couple of power stations on a lower hierarchical level. PSC1 to PSC5 are connected to transformers (T1 to T5) which distributes the produced energy over power lines, other transformers (T6 to T16), and switches (Sw1 to $\mathrm{Sw} 8)$ to the consumers ( $\mathrm{C} 1$ to $\mathrm{C} 10)$.

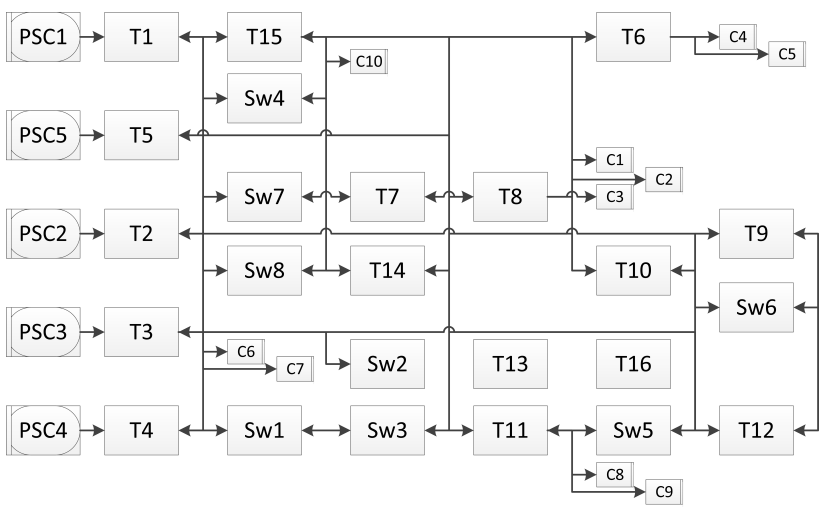

Figure 9. Real model of SEMSim Power.

Since the amount of commodities clearly outweighs the amount of processes, commodities are drawn bidirectional as described in Section 3.1. Excluding the consumers, all of the modeled entities together represent the power transmission system of Singapore, which is illustrated in Figure 10. 


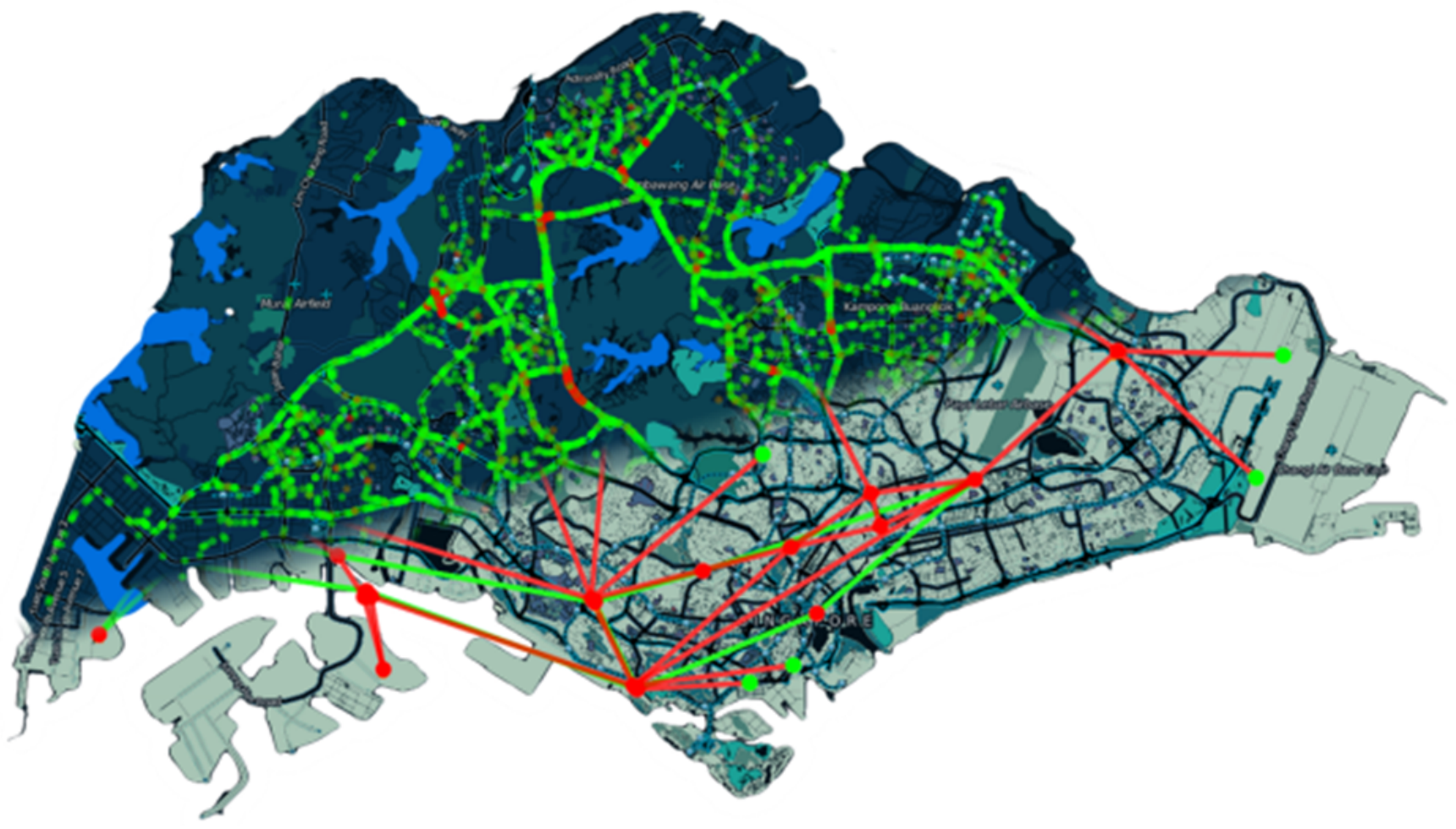

Figure 8. Overlapping visualisation of traffic simulated by SEMSim Traffic (upper half) and the power system simulated by SEMSim Power (bottom right half).

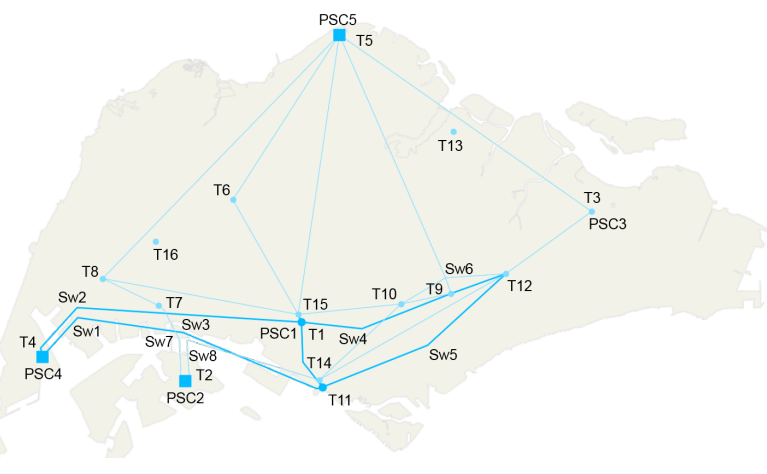

Figure 10. Power transmission system of Singapore.

\subsection{Data Model}

A data model explicitly describes the data structure of entity types, their attributes as well as their relationship types. These structures are derived from a specific real model. However, a data model may be used for many different real models, depending on how generic it is designed. The meta model of USES presented in Section 3.2 describes the most generic way a data model for energy system models should be based on. The data model of SEMSim Power is based on this meta model and was designed to facilitate the storage of all different kinds of energy system model data. However, this is only one possible implementation.
Beyond that, other data models based on the meta model of USES may be created. The data model is by default implemented in a relational database. In order to facilitate an easy exchange of data, XML files can also be used on the same schema.

Figure 11 shows the data model of SEMSim Power in the UML class diagram language. The four main entity types which are not owed to the process of database normalization [21] or building relationships are Process, Commodity, Model and Parameter.

- The entity types Process and Commodity in this model directly reflect the entity types Process and Commodity from the meta model of USES. Auxiliary related entity types are introduced to distinguish different types and groups of processes and commodities. Each process and each commodity comes with a location and a shape to be able to visualize the modeled energy system geographically and geometrically correct.

- The Model entity type provides a way to store data of different models or scenarios in the same database by allocating each process entity and each commodity entity to a unique model entity.

- The Parameter entity type and its directly related entity types are introduced to store all of the different kinds of parameters that may occur for entities within the modeled energy systems. Parameters are stored 


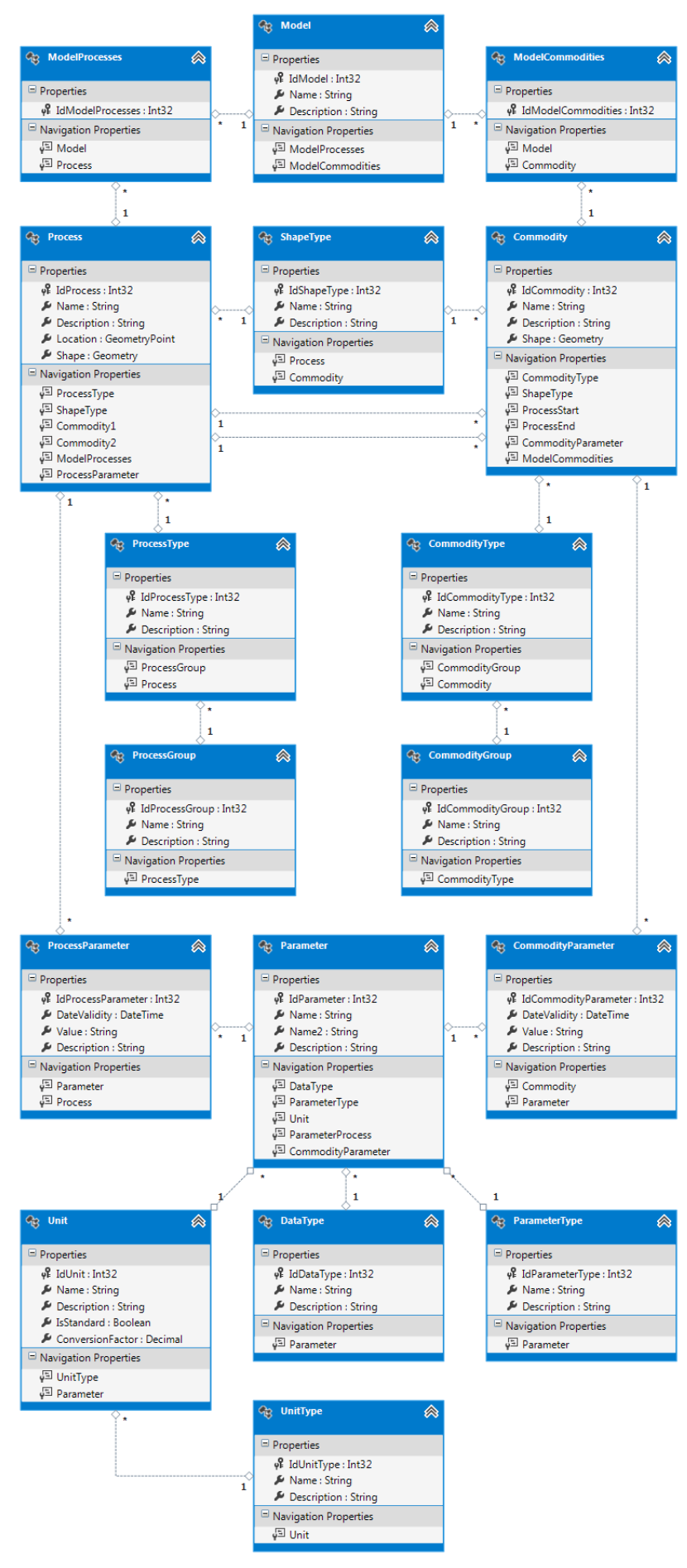

Figure 11. Data model of SEMSim Power.

in a specific unit of which all can be converted into another. Its values are process- and/or commodityrelated. Besides static values also time-dependent values can be provided for a parameter.

The actual power system simulation consists of the three process types Consumer (e.g., charging stations), Producer (e.g., power plants), and Prosumer (e.g., substations) and the commodity type Connection (e.g., power lines). Although the data model is country-independent SEMSim Power utilizes only data specific to Singapore at the moment. Data of other countries may be added upon availability. Because available data is scarce in Singapore only the transmission system with the maximum $(400 \mathrm{kV})$ and high $(230 \mathrm{kV})$ voltage grid is used ${ }^{10}$.

\subsection{Formal Model}

To include a behavior of the modeled entities a formal model that is based on the real model can be created using mathematical methods. With these methods a relaxed model of the real energy system is created with which only quantifiable objectives can be investigated. SEMSim Power uses the simulation method to calculate the energy flows and the behavior of the involved processes at any point in time and uses those numbers to determine thereby incurred costs.

The energy and power demand of all the consumers (i.e., charging stations), is obtained from the SEMSim Traffic simulation. As a result, the energy flows throughout the network can then be calculated during each time step of the distributed simulation (i.e., SEMSim Traffic and SEMSim Power are synchronized). For this purpose, an energy flow optimization using Ford-Fulkerson's maximum flow algorithm [22] (pp.334) in combination with Dijkstra's shortest path algorithm [22] (pp.329) is conducted.

\section{Conclusion and Future Work}

In this paper, we have introduced USES, the Universal Scheme for modeling Energy Systems. USES is a formal language and has been designed as an alternative for the outdated eRES. In addition, we have described an application example in the context of the electro-mobility research conducted at TUM CREATE in Singapore. Part of this research is to study the impact of city-wide use of electric vehicles on the power system. Gaining a better understanding of the impact of electro-mobility on the infrastructure is important for planning purposes. Since there is no case of a city where electric vehicles are used on a large scale ( $>100 \mathrm{k}$ vehicles), simulation is the only way to study such a system.

Exploratory simulation will be used to automatically explore a given scenario space by means of simulation. Each of these scenarios may be concerned with different configurations of the power system. For example, different scenarios may be concerned with different charging station placement or different charging technology (e.g., fast

\footnotetext{
${ }^{10}$ Except for consumers all of the used data originates from: http://www.ema.gov.sg/media/files/vesting_ contracts/10010665_CCGT_RMC_CalCs_Final.pdf 
charging vs. slow charging, wireless charging, etc.). In order to evaluate a large number of scenarios it is necessary to quickly create instances of the power system simulation that reflects the scenarios. Automatic generation of a simulation model is thus needed. USES can be used as a formal way of describing a simulation model that can then be instantiated automatically.

Future work will be concerned with synthetic generation of a more realistic power system (currently the model only includes the transmission system). This is necessary in order to obtain the required fidelity of the simulation model in order to study the tempo-spatial energy demand that will affect the power system. In addition, we will continue the development of USES in order to include additional features.

\section{Acknowledgement}

This work was financially supported by the Singapore National Research Foundation under its Campus for Research Excellence And Technological Enterprise (CREATE) program.

\section{References}

[1] K. Yasuda and T. Ishii. Decentralized Autonomous Control of Super Distributed Energy Systems. In 2002 IEEE International Conference on Systems, Man and Cybernetics, volume 6, pages 6-pp. IEEE, 2002.

[2] M. Blesl, A. Schweiker, and C. Schlenzig. Erweiterung der Analysemöglichkeiten von NetWork Der Netzwerkeditor. Technical Report 51, Institute for Energy Economics and the Rational Use of Energy, University Stuttgart, Germany, 1998.

[3] Y. Xu, H. Aydt, and M. Lees. SEMSim: A Distributed Architecture for Multi-scale Traffic Simulation. In Proceedings of the 2012 ACM/IEEE/SCS 26th Workshop on Principles of Advanced and Distributed Simulation, pages 178-180. IEEE Computer Society, 2012.

[4] M. Lu and C. Chen. The Design of Multi-agent based Distributed Energy Eystem. In IEEE International Conference on Systems, Man and Cybernetics, pages 2001-2006. IEEE, 2009.

[5] W. Palm. System Dynamics. McGraw-Hill Higher Education, 3rd edition, 2005.

[6] W. Middendorf. Design of Devices and Systems. CRC Press, 3rd edition, 1997.

[7] L. Krajewski, L. Ritzman, and M. Malhotra. Operations Management. Prentice Hall, 9th edition, 2009.
[8] X. Wang, Y. Song, and M. Irving. Modern power system analysis. Springer, NY, USA, 2008.

[9] C. Girault and R. Valk. Petri Nets for Systems Engineering: A Guide to Modeling, Verification, and Applications. Springer, 2003.

[10] W. Khalil, R. Merzouki, B. Ould-Bouamama, and H. Haffaf. Hypergraph Models for System of Systems Supervision Design. IEEE Transactions on Systems, Man and Cybernetics, Part A: Systems and Humans, 42(4):1005-1012, 2012.

[11] K. Hipel, M. Jamshidi, J. Tien, and C. White. The Future of Systems, Man, and Cybernetics: Application Domains and Research Methods. IEEE Transactions on Systems, Man, and Cybernetics, Part C: Applications and Reviews, 37(5):726-743, 2007.

[12] K. Hoffman. The United States Energy System - A Unified Planning Framework. PhD thesis, Polytechnic Institute of Brooklyn, New York, NY, USA, 1972.

[13] M. Beller. Reference Energy System Methodology. $\mathrm{PhD}$ thesis, 1976.

[14] A. Chakrabarti. Power System Dynamics and Simulation. PHI Learning Pvt. Ltd., Delhi, Indida, 2013.

[15] D. Chassin, K. Schneider, and C. Gerkensmeyer. GridLAB-D: An open-source power systems modeling and simulation environment. In Proceedings of the Transmission and Distribution Conference and Exposition, pages 1-5. IEEE, 2008.

[16] M. Zhou. Distributed Parallel Power System Simulation. In High Performance Computing in Power and Energy Systems, pages 71-100. Springer, 2013.

[17] F. Milano and L. Vanfretti. State of the Art and Future of OSS for Power Systems. In IEEE Power \& Energy Society General Meeting, pages 1-7. IEEE, 2009.

[18] M. Moffet, F. Sirois, and D. Beauvais. Review of Open Source Code Power Grid Simulation Tools for Long-term Parametric Simulations. Technical report, CanmetENERGY Technical Report 2011-137, 2011.

[19] R. Sebesta. Concepts of Programming Languages, volume 4. Addison Wesley, 10th edition, 2002.

[20] IEEE Standard for Modeling and Simulation (M\&S) High Level Architecture (HLA) - IEEE Std 15162000, 1516.1-2000, 1516.2-2000.

[21] C. Date. Database Design and Relational Theory: Normal Forms and All That Jazz. O'Reilly, CA, USA, 2012.

[22] M. Newman. Networks: An Introduction. Oxford University Press, NY, USA, 2009. 